\title{
Expression of TYMS in lymph node metastasis from low-grade glioma
}

\author{
BINGQIAN DING $^{1}$, MING GAO ${ }^{1}$, ZHENJIANG LI ${ }^{1}$, CHENYANG XU ${ }^{1}$, SHAOKANG FAN ${ }^{1}$ and WEIYA HE ${ }^{2}$ \\ Departments of ${ }^{1}$ Neurosurgery and ${ }^{2}$ Neurology, Huaihe Hospital of Henan University, Kaifeng, Henan 475000, P.R. China
}

Received September 4, 2014; Accepted May 26, 2015

DOI: $10.3892 / \mathrm{ol} .2015 .3419$

\begin{abstract}
The aim of the present study was to investigate the expression of thymidylate synthase (TYMS) in the primary foci and metastatic lymph nodes of low-grade glioma, and to analyze the function of TYMS in the lymph node metastases from low-grade glioma. The study included 93 cases of surgically resected and pathologically confirmed low-grade glioma, form patients treated at Huaihe Hospital of Henan University (Kaifeng, China). The following clinical data was obtained from each patient: Gender, age, subjective symptoms (dizziness, headache, a feeling of pressure in the head, etc.), site of disease, tumor type, pathological stage, degree of differentiation and lymph node involvement. The surgically resected gliomas and dissected cervical lymph nodes were immunohistochemically stained, and DNA was extracted from the tumor and lymph node tissues samples for polymerase chain reaction sequencing and amplification. The expression of TYMS in the primary foci and metastatic lymph nodes of low-grade glioma was examined. Additionally, the association between pathological features and the postoperative survival rate of the patients was analyzed. The primary lesions of all 93 cases exhibited positive TYMS expression and 43/157 (27.39\%) lymph nodes exhibited positive TYMS expression. Factors that significantly influenced the postoperative survival rate of the patients, included the metastasis of the cervical lymph nodes $(\mathrm{P}<0.01)$, the number of dissected cervical lymph nodes $(\mathrm{P}<0.01)$ and the degree of differentiation $(\mathrm{P}<0.05)$. The metastasis of the cervical lymph nodes was the only independent risk factor affecting postoperative disease-free survival. The risk of recurrence in patients with metastasis of the cervical lymph nodes was 6.3-fold higher than in those without metastasis $(\mathrm{P}<0.01)$. Thus, the results of the present study provide a theoretical basis for accurately predicting the prognosis of patients with low-grade malignant brain glioma,
\end{abstract}

Correspondence to: Dr Weiya He, Department of Neurology, Huaihe Hospital of Henan University, 8 Baobei Road, Kaifeng, Henan 475000, P.R. China

E-mail: weiyahe@163.com

Key words: brain glioma, thymidylate synthase, lymphatic metastasis, survival rate reducing the conjecture involved in selecting postoperative treatment strategies and improving therapeutic efficacy.

\section{Introduction}

Brain glioma, which originates from neuroglia cells, is the most common type of intracranial tumor, accounting for $\sim 45 \%$ of all cases. Worldwide, 20-50 million individuals succumb due to malignant glioma each year (1). While, in recent years, with the development of diagnostic instruments and numerous novel antitumor agents, the efficacy of glioma treatment has greatly improved, it remains unsatisfactory (2). A previous study reported that, even when patients with low-grade glioma are considered cured by surgery, $45 \%$ later succumbed, due to recurrence or metastasis of glioma within 2 years (3). This indicates that certain patients with low-grade gliomas that are considered to be completely resected, may have developed micrometastases prior to surgery (4). Often, the micrometastases cannot be located using conventional methods, such as imaging and histopathology, particularly in low-grade tumors. Therefore, investigation into the micrometastasis of low-grade glioma is important for improving the treatment and prognosis of patients with glioma (5).

The thymidylate synthase (TYMS) gene is a malignant tumor marker that was demonstrated to have high sensitivity and specificity for malignant metastasis in low-grade glioma (6). The present study detected the expression levels of the TYMS gene in low-grade malignant glioma tissues and adjacent lymph nodes, using immunohistochemical analysis. The current study aimed to provide novel recommendations for diagnosis, disease evaluation, treatment strategies and prognosis of low-grade malignant glioma, by exploring the association between TYMS gene expression in the primary foci and metastatic lymph nodes of low-grade malignant glioma, and to analyze the function of TYMS in the lymph node metastases of low-grade glioma.

\section{Patients and methods}

Patients. The present study included 93 patients with surgically resected and pathologically confirmed low-grade malignant glioma, who were treated at Huaihe Hospital of Henan University (Kaifeng, China) between July 2009 and January 2014. The following clinical data was obtained from each patient: Gender, age, subjective symptoms (dizziness, 
headache, a feeling of pressure in the head, etc.), disease site, tumor type, pathological stage, degree of differentiation and lymph node involvement.

According to the 2007 World Health Organization (WHO) classification (7), glioma is classified into four grades with respect to the malignancy of the glioma. Grade I glioma is pilocytic astrocytoma; grade II is low-grade malignant glioma; grade III is anaplastic astrocytoma; and grade IV is glioblastoma multiforme. WHO has also classified glioma as low-grade glioma, including general pathological classification of glioma grades I-II, and high-grade glioma, including grades III and IV glioma. Following treatment, the survival rate for patients with grade I or II glioma is good, with patients typically surviving a number of years.

The current cohort of 93 cases included 49 males and 44 females, with an age range of 23-72 years (mean age, $46.14 \pm 8.52$ years). According to the 2007 WHO classification criteria, the cohort included 28 cases of astrocytoma, 12 cases of oligodendroglioma, 10 cases of ependymoma, 11 cases of mixed glioma, 7 cases of choroid plexus tumor, 7 cases of mixed neuronal glial tumor, 12 cases of pineal parenchymal tumor and 6 cases of embryonal tumor. 15 patients with benign brain tumors [ 7 cases of meningioma, 6 cases of pituitary adenoma and 2 cases of acoustic neuroma] were also included in the study, comprising 9 males and 6 females, with an age range of 20-71 years (mean, 45.37 7.61 years). The present study was conducted in accordance with the declaration of Helsinki and approval was obtained from the Ethics Committee of Huaihe Hospital of Henan University. Written informed consent was obtained from all participants.

Immunohistochemistry. The surgically resected glioma specimens $(n=93)$ and dissected cervical lymph nodes $(n=157)$ were resected from the middle of the tissue in the longitudinal direction. These 250 samples were randomly marked by Arabic numerals from 1-250. The marked sample was resected from the middle of the tissue in the longitudinal direction and was further marked by A or B (e.g., 1A, 1B; 250A, 250B). Specimen A or B from the 250 samples was then randomly selected for pathological analysis.

The resected glioma specimens were formalin-fixed, paraffin-embedded and sliced into $4-\mu \mathrm{m}$ serial sections. The sections were dewaxed, hydrated and flushed with phosphate-buffered saline (PBS). Subsequently, polymer enhancer (Wuhan Boster Biological Technology, Ltd., Wuhan, China) and mouse anti-human TYMS monoclonal antibody (catalog no. CAB-4990MH; Creative BioMart, Shirley, NY, USA; dilution, 1:200) were applied. The sections were then incubated at room temperature for $1 \mathrm{~h}$ and washed with PBS. The secondary antibody was HRP-conjugated goat anti-mouse polyclonal antibody (catalog no. ab97023; Abcam, Cambridge, UK; dilution, 1:500). The sections were then incubated again at room temperature for $1 \mathrm{~h}$. Finally, diaminobenzidine chromogenic liquid (Daan Gene Co., Ltd.) was added. Following hematoxylin staining, the slices were sealed with neutral resin and the coloring process was performed using the immunohistochemical surfactant protein A method, according to the manufacturer's instructions (Beijing Dingguo Biotechnology Co. Ltd., Beijing, China). Positive and negative immunohistochemical controls were used for comparison, with PBS buffer solution used in place of the TYMS antibody as the negative control. Subsequent to specimen processing, the expression of TYMS in the glioma tissue and adjacent cervical lymph nodes was observed under a BX51 microscope (Olympus Corporation, Tokyo, Japan).

Polymerase chain reaction $(P C R)$ and reverse transcription-quantitative PCR (RT-qPCR). A MagNA Lyser Instrument (Roche Diagnostics, Basel, Switzerland) was used to homogenate the other half of the 157 lymph nodes and allow DNA extraction. A DNA sample $(1 \mu \mathrm{g})$ was selected for quality evaluation and concentration detection using a NanoDrop 2000 concentration detector (Thermo Fisher Scientific, Inc., Rockford, IL, USA). The samples were diluted to a working concentration of 20-40 ng/ $\mu 1$ and the TYMS gene was amplified by PCR with the following primers: TYMS forward, 5'-GGTATTGAGACAGACAGTGc-3'; TYMS reverse, 5'-CATGGGATCTGTTTCTTTGC-3'; $\beta$-actin (control) forward, 5'-GCGGGAAATCGTGCGTGAC-3'; and $\beta$-actin reverse, 5'-CGTCATACTCCTGCTTGCTG-3'. The primers were synthezised by Sangon Biotechnology Co. (Shanghai, China). Real-time quantitative PCR was performed using a SYBR Premix EX TaqTM kit (TaKaRa, Dalian, China), according to manufacturer's instructions. The PCR cycling conditions consisted of $95^{\circ} \mathrm{C}$ for $30 \mathrm{~s}$ followed by 35 cycles of denaturation at $95^{\circ} \mathrm{C}$ for $3 \mathrm{~s}$ and annealing at $60^{\circ} \mathrm{C}$ for $30 \mathrm{~s}$, extension at $72^{\circ} \mathrm{C}$ for $1 \mathrm{~min}$, and final extension at $72^{\circ} \mathrm{C}$ for $5 \mathrm{~min}$. The standard curve was then constructed, and the results were quantified using the $2^{-\Delta \Delta \mathrm{Ct}}$ method. The PCR products were purified and sequenced using an ABI 3130XL sequencer (Applied Biosystems, Foster City, CA, USA). In addition, the expression of TYMS mRNA in the lymph nodes was determined by performing RT-qPCR. The expression of the TYMS gene, as assessed by RT-qPCR, was used as marker with which to identify lymph node metastases. The associations between TYMS gene expression, and lymph node metastasis, clinical and pathological factors, postoperative survival and disease-free survival were also analyzed.

Evaluation criteria. Positive expression of the TYMS gene was defined as the observation of brown granules in the cytomembrane and/or cytoplasm. The intensity of the expression was determined by performing 10 high-magnification observations for every slice, and applying a semi-quantitative integral analysis method, according to the color intensity and percentage of positively stained cells. Staining intensity was quantified according to the number of the positive cells, as follows: $<5 \%$ positive cells, 0 points; $6-25 \%$ positive cells, 1 point; $25-50 \%$ positive cells, 2 points; $51-75 \%$ positive cells, 3 points; and $>75 \%$ positive cells, 4 points. The color intensity criterion was as follows: Dark brown, 3 points; light brown, 2 points; and light yellow, 1 point. Points from the two subcriteria were combined and the following overall scores were determined: 6-7 points, strong positive $(+++)$; $4-5$ points, moderate positive $(++) ; 2-3$ points, weak positive $(+)$; and $0-1$ points, negative (-) (8).

Association between the expression of TYMS and relevant factors in patients with glioma. In accordance with a previous 
A

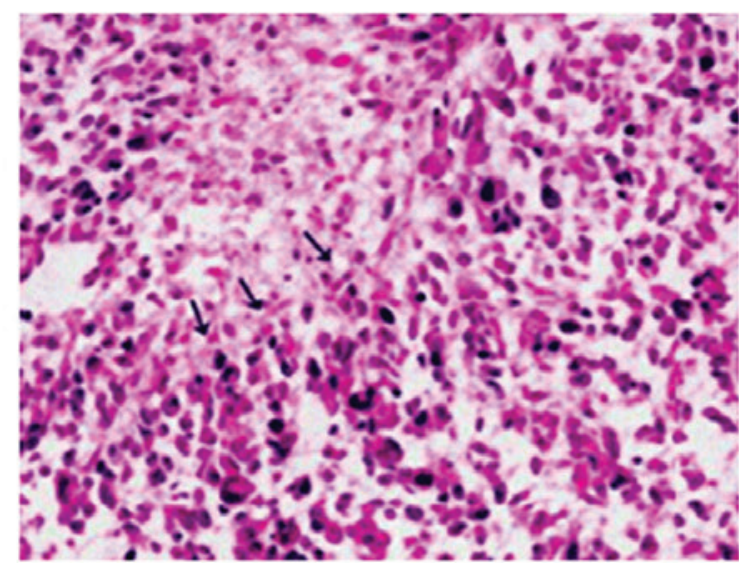

B

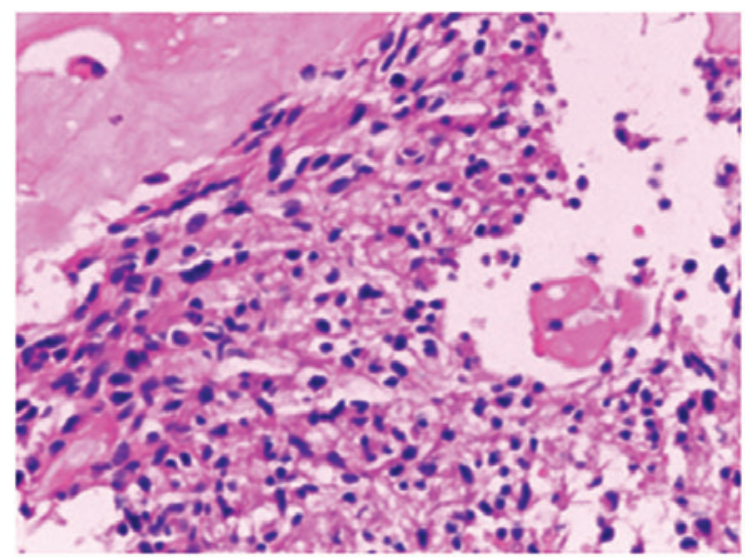

C

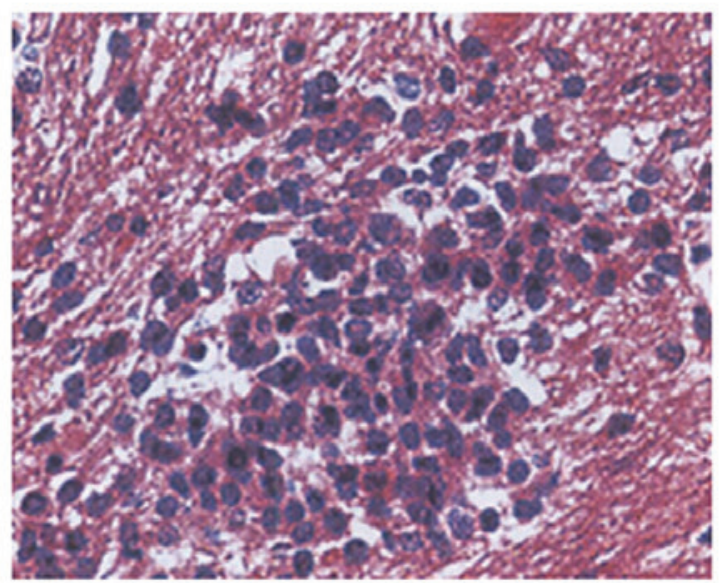

D

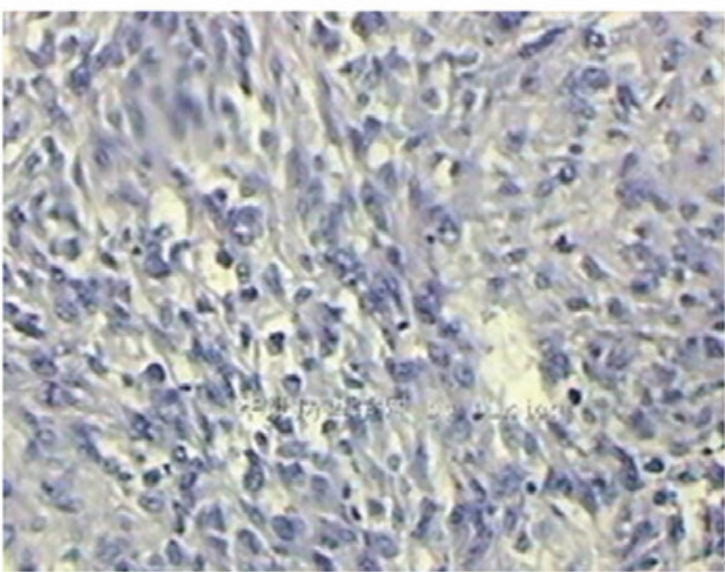

Figure 1. Positive expression of thymidylate synthase in (A) and (B) hematoxylin and eosin; and (C) and (D) hematoxylin-stained immunohistochemical samples from patients with low-grade malignant glioma (magnification, x200). study (9), data on gender, age, subjective symptoms, disease site, tumor type, pathological stage, degree of differentiation and metastasis of the lymph nodes were collected from all current patients exhibiting low-grade malignant brain glioma. The present study used statistics to analyze the association between lymph node metastasis and these factors.

Statistical analysis. Statistical analysis was performed using SPSS software (version 14.0; SPSS Inc., Chicago, IL, USA). Each parameter was split into two categories, which were compared by performing a t-test. The associations between lymph node metastasis of low-grade primary malignant glioma tumors and other factors were analyzed using logistic regression analysis. Furthermore, the expression of the TYMS gene and the postoperative survival rate were analyzed using Kaplan-Meier survival analysis, and independent risk factors were analysis using Cox regression analysis. $\mathrm{P}<0.05$ was considered to indicate a statistically significant difference.

\section{Results}

TYMS protein expression and lymph node metastasis. In the 15 patients with benign brain tumors, TYMS protein expression was negative in all cervical lymph nodes examined. However, for the 93 patients with low-grade malignant glioma, TYMS protein expression was positive in all primary lesions (100\%) and in 43/157 (27.93\%) lymph nodes, as indicated in Fig. 1.

Analysis of the postoperative overall survival rate. Relevant clinical factors and lymph node metastasis were analyzed using Kaplan-Meier survival analysis. The results indicated that cervical lymph node metastasis $(\mathrm{P}<0.01)$, the number of dissected cervical lymph nodes $(\mathrm{P}<0.05)$ and the degree of differentiation $(\mathrm{P}<0.05)$ were significant factors influencing postoperative survival. However, gender, age, subjective symptoms, disease site, tumor type and pathological stage exhibited a limited association with the survival rate of the patients (Table I).

Additional analysis of the association between metastases to cervical lymph nodes and the postoperative survival rate, identified that the survival rate of patients with cervical lymph node metastasis was 6.3-fold higher than in those without cervical lymph node metastasis $(\mathrm{P}<0.01$; data not shown). Furthermore, the greater the number of lymph nodes dissected, the higher the patient mortality.

Analysis of the postoperative disease-free survival rate. The same method was applied in order to analyze the factors associated the postoperative disease-free survival rate. The following factors were demonstrated to be significantly correlated with disease-free survival, using Kaplan-Meier survival analysis: Metastasis to the cervical lymph nodes $(\mathrm{P}<0.01)$, the number of dissected cervical lymph nodes $(\mathrm{P}<0.05)$, the degree of differentiation $(\mathrm{P}<0.05)$ and the presence of subjective symptoms $(\mathrm{P}<0.05$; Table II). Additional analysis of the association between metastases to the cervical lymph nodes and postoperative disease-free survival, demonstrated that the mortality of patients with metastasis to the cervical lymph 
Table I. Association between postoperative overall survival rate and other factors in patients with low-grade malignant glioma.

\begin{tabular}{|c|c|c|c|c|}
\hline \multirow[b]{2}{*}{ Factor } & \multicolumn{2}{|c|}{ Category } & \multirow[b]{2}{*}{$\chi^{2}$} & \multirow[b]{2}{*}{ P-value } \\
\hline & 1 & 2 & & \\
\hline Gender & Male & Female & 0.481 & 0.539 \\
\hline Age, years & $<60$ & $\geq 60$ & 0.164 & 0.738 \\
\hline Subjective symptoms & No & Yes & 2.922 & 0.079 \\
\hline Disease site & Cerebral hemisphere & Cerebellum & 0.897 & 0.348 \\
\hline Tumor type & Astrocytoma & Oligodendroglioma & 0.189 & 0.718 \\
\hline Pathological stage & Ia & $\mathrm{Ib}$ & 0.849 & 0.361 \\
\hline Degree of differentiation & Low & High & 4.875 & $0.041^{\mathrm{a}}$ \\
\hline Metastasis of cervical lymph nodes ${ }^{b}$ & No & Yes & 13.133 & $<0.001^{\mathrm{a}}$ \\
\hline Dissected cervical lymph nodes, $\mathrm{n}$ & $<5$ & $\geq 5$ & 6.693 & $0.022^{\mathrm{a}}$ \\
\hline
\end{tabular}

${ }^{\mathrm{a}} \mathrm{P}<0.05$; ${ }^{\mathrm{b}}$ according to thymidylate synthase mRNA expression.

Table II. Association between postoperative disease-free survival rate and other factors in patients with low-grade malignant glioma.

\begin{tabular}{|c|c|c|c|c|}
\hline \multirow[b]{2}{*}{ Factor } & \multicolumn{2}{|c|}{ Category } & \multirow[b]{2}{*}{$\chi^{2}$} & \multirow[b]{2}{*}{ P-value } \\
\hline & 1 & 2 & & \\
\hline Gender & Male & Female & 0.514 & 0.492 \\
\hline Age, years & $<60$ & $\geq 60$ & 0.128 & 0.780 \\
\hline Subjective symptoms & No & Yes & 4.975 & $0.040^{\mathrm{a}}$ \\
\hline Disease site & Cerebral hemisphere & Cerebellum & 0.833 & 0.371 \\
\hline Tumor type & Astrocytoma & Oligodendroglioma & 0.328 & 0.609 \\
\hline Pathological stage & $\mathrm{Ia}$ & $\mathrm{Ib}$ & 2.691 & 0.102 \\
\hline Degree of differentiation & Low & High & 5.693 & $0.032^{\mathrm{a}}$ \\
\hline Metastasis of cervical lymph nodes ${ }^{b}$ & No & Yes & 18.268 & $<0.001^{\mathrm{a}}$ \\
\hline Dissected cervical lymph nodes, $\mathrm{n}$ & $<5$ & $\geq 5$ & 11.482 & $0.002^{\mathrm{a}}$ \\
\hline
\end{tabular}

${ }^{\mathrm{a}} \mathrm{P}<0.05$; ${ }^{\mathrm{b}}$ according to thymidylate synthase mRNA expression.

nodes was 8.4-fold higher than that in patients without metastasis $(\mathrm{P}<0.01$; data not shown).

Independent risk factors affecting the postoperative disease-free survival rate. The following factors were substituted into the Cox regression analysis: Gender, age, disease site, tumor type, pathological stage, lymph node metastasis, cervical lymph node metastasis and number of dissected lymph nodes. The results indicated that metastasis to the cervical lymph nodes was the only independent risk factor affecting postoperative disease-free survival. The risk of recurrence in patients with metastasis to the cervical lymph nodes was 7.3-fold higher than that in those without metastasis $(\mathrm{P}<0.01$; data not shown).

Follow-up. The patient was followed-up for 3-65 months until December 2013, with a median follow-up time of 37 months. A total of 93 patients dropped out of the follow-up process, resulting in a dropout rate of $5.38 \%$. It was noted that 28 patients $(30.11 \%)$ did not exhibit recurrence or metastasis until after the follow-up process had ended; 9 patients $(9.68 \%)$ experienced recurrence or metastasis, but remained alive; and 51 patients $(54.84 \%)$ succumbed due to disease progression during the follow-up period.

\section{Discussion}

Brain glioma is a tumor type that continues to be associated with a poor prognosis. It is well known that the likelihood of curing glioma is closely associated with disease stage, in addition to the methods of diagnosis and treatment. Early detection is critical to improving the therapeutic effect and increasing the survival of patients with glioma (10). In recent years, with the development of novel medical technologies, the early diagnosis and rate of successful treatment of low-grade malignant glioma have significantly improved. However, the overall survival rate of patients with glioma remains unsatisfactory. Therefore, there has been increased interest in improving the therapeutic response of glioma, particularly malignant glioma. In addition, the molecular mechanism underlying the 
pathogenesis of glioma, as well as novel therapeutic approaches for glioma, have been explored in recent years (11). A previous clinical study demonstrated that the same type of glioma exhibited significant differences in treatment effect, prognosis, invasion, recurrence and metastasis (12). In the current study, molecular biology and immunohistochemistry technologies were employed to explore the role of TYMS genes in gliomas, which may provide valuable guidance for future treatment and prognosis evaluation.

The 5-year survival rate of patients with low-grade malignant glioma is not satisfactory, possibly due to micrometastasis to lymph nodes, blood or bone marrow (13). These micrometastatic deposits may be a major cause of recurrence in glioma. Micrometastases are difficult to detect using conventional methods, such as imaging and clinical pathology (14). The malignant tumor cells of micrometastatic deposits may spread and survive in the lymphatic circulatory system, blood, bone marrow, various tissues and organs, resulting in numerous relevant clinical manifestations (15).

A number of preliminary studies have demonstrated that differences in treatment effect, prognosis, invasion, recurrence and metastasis among the same pathological type of glioma may be caused by differences in the expression levels of specific genes. Therefore, it is important to detect any changes in such genes and associated proteins, using molecular biology and immunohistochemical techniques $(16,17)$. Clinically, optimal and individualized treatment regimes may be designed for each individual patient with glioma, according to the results of genetic tests, clinical data, surgery, pathological analysis and diagnosis (18). Certain studies have demonstrated that TYMS has high specificity and sensitivity in detecting low-grade malignant metastasis of glioma. TYMS may be specifically expressed in glioma tissues, while is not expressed in normal lymph nodes, indicating that TYMS may be useful as a marker with which to identify lymph node metastasis. Therefore, detecting TYMS expression in low-grade malignant lymph node metastases of glioma may enable a more accurate determination of prognosis in patients with glioma (19).

The TYMS gene is located on the short arm of chromosome 18 and encodes TYMS, a key enzyme involved in DNA synthesis. TYMS is important for DNA synthesis and repair, and is critical in folate metabolism circulation. TYMS is also currently an important target enzyme in clinical chemotherapy based on 5-fluorouracil (5-FU) (20). Metabolites of 5-fluorodeoxyuridine monophosphate bind to TYMS, blocking the function of TYMS and thereby inhibiting DNA synthesis. Numerous clinical studies in colorectal, lung, breast, head and neck squamous cell carcinoma, as well as other types of tumor, have identified that TYMS mRNA expression levels are closely associated with the effects of 5-FU-based chemotherapy. In addition, TYMS has an important role in the individualized treatment of glioma, as its presence helps to predict patient sensitivity to radiotherapy and chemotherapy. Previous clinical studies have demonstrated that patients with low levels of TYMS mRNA expression exhibit good responses to 5-FU chemotherapy $(21,22)$. By contrast, patients with high TYMS expression exhibit poor responses to fluoride chemotherapy. Furthermore, a previous study identified that the efficacy of pemetrexed, a novel antifolate agent, was negatively correlated with the expression level of TYMS mRNA (23).
In the present study, data regarding gender, age, subjective symptoms, disease site, tumor type, degree of differentiation, metastasis to cervical lymph nodes and the number of dissected lymph nodes, were collected from patients with low-grade malignant brain glioma. It was identified that lymph node metastasis with TYMS mRNA expression had no significant correlation with gender, age, smoking, pathological type and pathological grade. However, lymph node metastasis was closely associated with the number of dissected lymph nodes and the degree of differentiation. Following metastasis to neck lymph nodes, prognosis is poor and the postoperative survival time is short. The greater the number of dissected lymph nodes, the lower the relative probability of tumor metastasis and recurrence following surgery. In addition, the lower the degree of tumor differentiation, the higher the degree of malignancy, the poorer the prognosis, and the shorter the postoperative survival time.

Following clarification of the associated risk factors, the present study analyzed the association between the aforementioned factors and disease-free survival of postoperative patients. The results of the Kaplan-Meier survival analysis indicated that the factors affecting postoperative survival included cervical lymph node metastasis, the number of lymph nodes dissected and the degree of differentiation $(\mathrm{P}<0.05)$. Additional analysis of the association between disease-free survival and lymph node metastasis of the neck, identified that the risk of mortality was 28.4-fold higher in patients with cervical lymph node metastasis compared with those without cervical lymph node metastasis $(\mathrm{P}<0.01)$.

Although the sensitivity of RT-qPCR technology is significantly greater than that of routine pathological examination, the process of metastasis of low-grade malignant glial neoplasm is complex and its underlying mechanisms remain unclear. Furthermore, detection of abnormal expression of TYMS will not identify every lymph node metastasis. Therefore, additional investigation of the molecular mechanism underlying lymph node metastasis is required, in order to provide a theoretical basis and empirical evidence for the accurate staging of low-grade malignant glioma, as well as informing multidisciplinary treatment strategies for postoperative patients (24).

Due to the unique nature and diversity of gliomas, the implementation of individualized therapy and targeted drug treatment is the optimal clinical treatment strategy for clinicians and patients (25). Adjuvant therapies applied prior to surgery to patients with low-grade malignant glioma, may reduce tumor size and decrease tumor stage, allowing certain patients with locally advanced disease to undergo surgical treatment. Detection of lymph node metastasis provides a basis for more accurate staging of low-grade malignant glioma and guides treatment selection (26). In the present study, the association between TYMS gene expression and the prognosis of patients with low-grade malignant glioma was investigated by detecting the expression of the TYMS gene in primary tumors and adjacent lymph nodes. The results of the present study may facilitate the accurate prediction of the prognosis of patients with low-grade malignant brain glioma, lowering invasive intervention, reducing the conjecture involved in selecting postoperative treatment strategies and improve therapeutic efficacy (27). 
The association between TYMS expression, and highly malignant glioma and lymph nodes metastasis has been investigated in previous studies. However, to the best of our knowledge, studies analyzing the correlation between these factors are absent from the literature. The present study observed the expression of TYMS in low-grade malignant glioma tissue and adjacent lymph nodes, and analyzed the correlation between TYMS expression, and low-grade malignant glioma, lymph node metastasis, clinicopathological factors and postoperative survival rate. These results may be useful in guiding the clinical diagnosis, evaluations, treatment and prognosis of patients with low-grade malignant glioma. Due to the abnormal expression of TYMS in the lymph nodes adjacent to the low-grade malignant glioma, TYMS expression is hypothesized to be an indicator of lymph node metastasis. The present study demonstrated that TYMS expression is significantly correlated with overall and disease-free survival of patients with low-grade malignant glioma. Therefore, certain high-risk patient subgroups may be identifiable by detection of abnormal TYMS expression in lymph nodes adjacent to the low-grade malignant brain glioma lesion. In addition, this data may facilitate the accurate staging of disease, allowing certain patients with low-grade malignant brain glioma micrometastases to be more easily identified and, thus, to undergo earlier intervention. Future studies should determine the most comprehensive treatment strategy for low-grade malignant brain glioma with lymph node metastasis in order to improve the long-term survival rates of such patients.

\section{References}

1. Stupp R, Hegi ME, Mason WP, van den Bent MJ, Taphoorn MJ, Janzer RC, Ludwin SK, Allgeier A, Fisher B, Belanger K, et al: Effects of radiotherapy with concomitant and adjuvant temozolomide versus radiotherapy alone on survival in glioblastoma in a randomised phase III study: 5-year analysis of the EORTC-NCIC trial. Lancet Oncol 10: 459-466, 2009.

2. Sanai N, Chang S and Berger MS: Low-grade gliomas in adults. J Neurosurg 115: 948-965, 2011.

3. Stewart LA: Chemotherapy in adult high-grade glioma: A systematic review and meta-analysis of individual patient data from 12 randomised trials. Lancet 359: 1011-1018, 2002.

4. Athanassiou H, Synodinou M, Maragoudakis E, Paraskevaidis M, Verigos C, Misailidou D, Antonadou D, Saris G, Beroukas K and Karageorgis P: Randomised phase II study of temozolomide and radiotherapy compared with radiotherapy alone in newly diagnosed glioblastoma multiforme. J Clin Oncol 23 : 2372-2377, 2005.

5. Mason WP, Maestro RD, Eisenstat D, Forsyth P, Fulton D, Laperrière N, Macdonald D, Perry $\mathrm{J}$ and Thiessen B; Canadian GBM Recommendations Committee: Canadian recommendations for the treatment of glioblastoma multiforme. Curr Oncol 14: 110-117, 2007.

6. Masuda TA, Inoue H, Sonoda H, Mine S, Yoshikawa $\mathrm{Y}$, Nakayama K, Nakayama K and Mori M: Clinical and biological significance of S-phase kinase-associated protein 2 (Skp2) gene expression in gastric carcinoma: Modulation of malignant phenotype by Skp2 overexpression, possibly via p27 proteolysis. Cancer Res 62: 3819-3825, 2002.

7. Rosenblum MK: The 2007 WHO Classification of Nervous System Tumors: Newly recognized members of the mixed glioneuronal group. Brain Pathol 17: 308-313, 2007.

8. Weller M: Management of gliomas: Relevance of molecular markers for clinical practice. Eur Assoc Neuro Oncol Magazine 2: 6-10, 2012.

9. Shaw E, Arusell R, Scheithauer B, et al: Prospective randomized trial of low-versus high-dose radiation therapy in adults with supratentorial low-grade glioma: Initial report of a North Central Cancer Treatment Group/Radiation Therapy Oncology Group/Eastern Cooperative Oncology Group study. J Clin Oncol 20: 2267-2276, 2002.
10. Stupp R, Mason WP, van den Bent MJ, Weller M, Fisher B, Taphoorn MJ, Belanger K, Brandes AA, Marosi C, Bogdahn U, et al: Radiotherapy plus concomitant and adjuvant temozolomide for glioblastoma. N Engl J Med 352: 987-996, 2005.

11. Takanami I: The prognostic value of overexpression of Skp2 mRNA in non-small cell lung cancer. Oncol Rep 13: 727-731, 2005.

12. Kamata Y, Watanabe J, Nishimura Y, Arai T, Kawaguchi M, Hattori M, Obokata A and Kuramoto H: High expression of skp2 correlates with poor prognosis in endometrial endometrioid adenocarcinoma. J Cancer Res Clin Oncol 131: 591-596, 2005.

13. Pedeutour-Braccini Z, Burel-Vandenbos F, Gozé C, Roger C, Bazin A, Costes-Martineau V, Duffau H and Rigau V: Microfoci of malignant progression in diffuse low-grade gliomas: Towards the creation of an intermediate grade in glioma classification? Virchows Arch 466: 433-444, 2015.

14. Westhoff MA, Zhou S, Nonnenmacher L, Karpel-Massler G, Jennewein C, Schneider M, Halatsch ME, Carragher NO, Baumann B, Krause A, et al: Inhibition of NF- $\kappa \mathrm{B}$ signaling ablates the invasive phenotype of glioblastoma. Mol Cancer Res 11: 1611-1623, 2013.

15. Yang G, Ayala G, De Marzo A, Tian W, Frolov A, Wheeler TM, Thompson TC and Harper JW: Elevated Skp2 protein expression in human prostate cancer: Association with loss of the cyclin-dependent kinase inhibitor p27 and PTEN and with reduced recurrence-free survival. Clin Cancer Res 8: 3419-3426, 2002.

16. Ezoe S, Matsumura I, Nakata S, Gale K, Ishihara K, Minegishi N, Machii T, Kitamura T, Yamamoto M, Enver T, et al: GATA-2/estrogen receptor chimera regulates cytokine-dependent growth of hematopoietic cells through accumulation of p21 (WAF1) and p27 (Kip1) proteins. Blood 100: 3512-3520, 2002.

17. Kudo Y, Kitajima S, Sato S, Miyauchi M, Ogawa I and Takata T: High expression of S-phase kinase-interacting protein 2, human F-box protein, correlates with poor prognosis in oral squamous cell carcinomas. Cancer Res 61: 7044-7047, 2001.

18. Van Meir EG, Hadjipanayis CG, Norden AD, Shu HK, Wen PY and Olson J: Exciting new advances in neuro-oncology: The avenue to a cure for malignant glioma. CA Cancer J Clin 60: 166-193, 2010.

19. Mamillapalli R, Gavrilova N, Mihaylova VT, Tsvetkov LM, Wu H, Zhang $\mathrm{H}$ and Sun H: PTEN regulates the ubiquitin-dependent degradation of the CDK inhibitor p27 (KIP1) through the ubiquitin E3 ligase SCF (SKP2). Curr Biol 11: 263-267, 2001.

20. Dong Y, Sui L, Watanabe Y, Sugimoto K and Tokuda M: S-phase kinase-associated protein 2 expression in laryngeal squamous cell carcinomas and its prognostic implications. Oncol Rep 10: 321-325, 2003.

21. Koumarianou A, Tzeveleki I, Mekras D, Eleftheraki AG, Bobos M, Wirtz R, Fountzilas E, Valavanis C, Xanthakis I, Kalogeras KT, et al: Prognostic markers in early-stage colorectal cancer: Significance of TYMS mRNA expression. Anticancer Res 34: 4949-4962, 2014.

22. Gotanda K, Hirota T, Matsumoto N and Ieiri I: MicroRNA-433 negatively regulates the expression of thymidylate synthase (TYMS) responsible for 5-fluorouracil sensitivity in HeLa cells. BMC Cancer 13: 369, 2013.

23. von der Lehr N, Johansson S, Wu S, Bahram F, Castell A, Cetinkaya C, Hydbring P, Weidung I, Nakayama K, Nakayama KI, et al: The F-box protein Skp2 participates in c-Myc proteosomal degradation and acts as a cofactor for c-Myc-regulated transcription. Mol Cell 11: 1189-1200, 2003.

24. Latres E, Chiarle R, Schulman BA, Pavletich NP, Pellicer A, Inghirami $G$ and Pagano M: Role of the F-box protein Skp2 in lymphomagenesis. Proc Natl Acad Sci USA 98: 2515-2520, 2001.

25. Qin L, Wang CZ, Fan HJ, Zhang CJ, Zhang HW, Lv MH and Cui SD: A dual-targeting liposome conjugated with transferrin and arginine-glycine-aspartic acid peptide for glioma-targeting therapy. Oncol Lett 8: 2000-2006, 2014.

26. Hua W, Yao Y, Chu YW, Zhong P, Zhang R, Zhu W, Wu JS, Ma DX, Xu M, Mao Y and Zhou LF: Phase I study of dendritic cells pulsed with tumor stem-like cells associated antigens against malignant glioma in recurrent patients. Chin J Neurosurg 27: 90-93, 2011.

27. Hart MG, Grant R, Garside R, Rogers G, Somerville M and Stein K: Temozolomide for high grade glioma. Cochrane Database Syst Rev 8: CD007415, 2008. 